\title{
Non-invasive terahertz field imaging inside parallel plate waveguides
}

Iwaszczuk, Krzysztof; Andryieuski, Andrei; Lavrinenko, Andrei; Zhang, X. -C.; Jepsen, Peter Uhd

Published in:

Applied Physics Letters

Link to article, DOI:

$10.1063 / 1.3628340$

Publication date:

2011

Document Version

Publisher's PDF, also known as Version of record

Link back to DTU Orbit

Citation (APA):

Iwaszczuk, K., Andryieuski, A., Lavrinenko, A., Zhang, X. -C., \& Jepsen, P. U. (2011). Non-invasive terahertz field imaging inside parallel plate waveguides. Applied Physics Letters, 99(7), 071113.

https://doi.org/10.1063/1.3628340

\section{General rights}

Copyright and moral rights for the publications made accessible in the public portal are retained by the authors and/or other copyright owners and it is a condition of accessing publications that users recognise and abide by the legal requirements associated with these rights.

- Users may download and print one copy of any publication from the public portal for the purpose of private study or research.

- You may not further distribute the material or use it for any profit-making activity or commercial gain

- You may freely distribute the URL identifying the publication in the public portal

If you believe that this document breaches copyright please contact us providing details, and we will remove access to the work immediately and investigate your claim 


\title{
Non-invasive terahertz field imaging inside parallel plate waveguides
}

\author{
K. Iwaszczuk, ${ }^{1, a)}$ A. Andryieuski, ${ }^{1}$ A. Lavrinenko, ${ }^{1}$ X.-C. Zhang, ${ }^{2}$ and P. U. Jepsen ${ }^{1}$ \\ ${ }^{1}$ DTU Fotonik, Technical University of Denmark, DK-2800 Kgs. Lyngby, Denmark \\ ${ }^{2}$ Center for Terahertz Research, Rensselaer Polytechnic Institute, Troy, New York 12180, USA
}

(Received 11 July 2011; accepted 4 August 2011; published online 19 August 2011)

\begin{abstract}
We present a non-invasive broadband air photonic method of imaging of the electric field of $\mathrm{THz}$ pulses propagating inside a tapered parallel plate waveguide. The method is based on field-enhanced second harmonic generation of the fundamental laser beam in an external electric field. We apply the technique to measure the frequency-dependent reflection coefficient at the end of the waveguide. (c) 2011 American Institute of Physics. [doi:10.1063/1.3628340]
\end{abstract}

Guiding of terahertz (THz) waves by various structures is a topic of extensive investigation. ${ }^{1}$ Due to its unique properties such as dispersionless propagation of the TEM mode with no frequency cut-off, the parallel plate waveguide (PPWG) has attracted significant attention. ${ }^{2-5}$ Recently, a tapered parallel plate waveguide (TPPWG) has been proposed. ${ }^{6-8} \mathrm{THz}$ waves can be confined inside a TPPWG on subwavelength scale in both transverse dimensions, ${ }^{9}$ thus enabling $\mathrm{THz}$ near-field spectroscopy with subwavelength resolution. The TPPWG also offers significant field enhancement and, thus, has a big potential for investigation of nonlinear $\mathrm{THz}$ phenomena with tabletop laser sources, which in recent years has become a very active research field. For experimental characterization of the TPPWG, it is necessary to characterize the electric field distribution inside the waveguide. Recently, a method based on scattering of the field from needle tip inserted into the waveguide was demonstrated. ${ }^{10}$

In this paper, we present a non-invasive method for characterization of the propagating electric field inside a TPPWG. We adapt the air bias coherent detection (ABCD) technique ${ }^{11}$ and apply it to a TPPWG in order to image the $\mathrm{THz}$ electric field distribution inside the waveguide along the propagation direction without disturbing the propagation of the guided $\mathrm{THz}$ wave. As an application of the method, we demonstrate the direct measurement of the reflection coefficient for the $\mathrm{THz}$ wave at the end of the waveguide.

Figure 1 shows the schematic of the experimental setup. The TPPWG consists of two electrically isolated, fine polished aluminum plates of a varying width (input $3 \mathrm{~mm}$, output $49 \mu \mathrm{m}$ ) and a varying plate separation (input $1 \mathrm{~mm}$, output $200 \mu \mathrm{m}$ ). The total length of the TTPWG is $25.4 \mathrm{~mm}$. A high voltage modulator (HVM) delivering $\pm 100 \mathrm{~V}$ at 500 $\mathrm{Hz}$ (synchronized with first subharmonic of the laser repetition rate) is connected to the waveguide plates. The applied voltage results in an electric bias field of $\pm 5 \mathrm{kV} / \mathrm{cm}$ at the point of the narrowest gap between metal plates. Broadband $\mathrm{THz}$ pulses generated by tilted pulsefront optical rectification in $\mathrm{LiNbO}_{3}$ (Ref. 12) are coupled into the TPPWG at the wide end of the waveguide. The $\mathrm{THz}$ wave propagates between aluminum plates in a TEM mode and then couples out into the free space at the narrow end of the waveguide. A

\footnotetext{
${ }^{a)}$ Electronic mail: kiwa@fotonik.dtu.dk.
}

near-infrared (NIR) beam from a $1 \mathrm{kHz}, 90 \mathrm{fs}, 800 \mathrm{~nm}$ regenerative Ti:sapphire femtosecond laser amplifier (SpectraPhysics Hurricane) is used for probing the $\mathrm{THz}$ field between the plates of the waveguide. The NIR beam is focused in the center of the volume between the plates with a $19 \mathrm{~mm}$-focal length lens (L1) and recollimated after the waveguide with lens L2. The beam waist at the focus point has been estimated to be $6.5 \mu \mathrm{m}$ and the Rayleigh range is $41 \mu \mathrm{m}$. The highest NIR pulse energy limited to $18 \mu \mathrm{J}$ in order to avoid ionization of the air. This part of the setup is mounted on a translation stage that enables scanning along the waveguide ( $z$ direction). The beam is sent through $400 \mathrm{~nm}$ bandpass filters to a photomultiplier tube (PMT) which detects the 400$\mathrm{nm}$ light generated in the ABCD process. The signal from the PMT is measured by a lock-in amplifier referenced to the HVM modulation frequency. The polarizations of the four interacting electric fields (terahertz, fundamental, second harmonic (SH), and bias) are perpendicular to the waveguide plates.

Coherent detection of $\mathrm{THz}$ radiation is based on the interaction between the laser pulse $I_{\omega}$ and the $\mathrm{THz}$ electric field $E_{T H z}$ in the presence of the external oscillating electric field $E_{\text {bias }}$. According to Karpowicz et al., ${ }^{11}$ the intensity $I_{2 \omega}(\vec{r}, t)$ of the $\mathrm{SH}$ generated at the point $\vec{r}$ and time $t$ as a result of such an interaction can be expressed by

$$
I_{2 \omega}(\vec{r}, t) \propto 4\left[\chi^{(3)} I_{\omega}(\vec{r}, t)\right]^{2} E_{\text {bias }}(\vec{r}, t) E_{T H z}(\vec{r}, t),
$$

where $\chi^{(3)}$ is the third-order nonlinear susceptibility of the gas. We assume that $E_{\text {bias }}$ is constant during the interaction time between the THz transient and the probe pulse.

In the standard $\mathrm{ABCD}$ configuration, the probe pulse and the $\mathrm{THz}$ transient propagate collinearly through the area of a bias field. For a given THz-probe delay time $\tau$, each point of the NIR probe interacts with the same part of a $\mathrm{THz}$ waveform through out the whole interaction length. Thus, the detected SH intensity $I_{2 \omega}(\tau)$ is proportional to the time convolution between the $\mathrm{THz}$ waveform $E_{T H z}(t)$ and the square of probe intensity $I_{\omega}^{2}(t)$,

$$
I_{2 \omega}(\tau) \propto E_{\text {bias }} \int E_{T H z}(t) I_{\omega}^{2}(\tau-t) d t .
$$

In our configuration, the $\mathrm{THz}$ transient and the probe pulse propagate in perpendicular directions. In this case for a given 


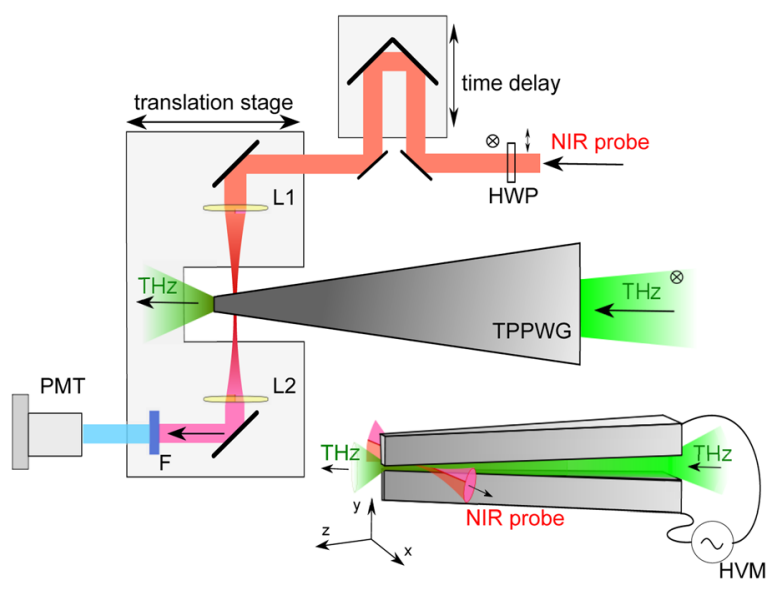

FIG. 1. (Color online) Schematic of the air photonic setup for non-invasive field imaging inside a tapered parallel plate waveguide. $\mathrm{THz}$ radiation is coupled into the TPPWG, propagates along it, and then interacts with NIR probe inducing second harmonic generation in the presence of oscillating local electric bias field. L1, L2-lenses, F-400 nm filter, HVM-high voltage modulator, and PMT—photomultiplying tube.

THz-probe delay time $\tau$, each point of the NIR probe interacts with a moving $\mathrm{THz}$ waveform and the intensity $I_{2 \omega}(\tau)$ of the generated $\mathrm{SH}$ is expressed as

$$
I_{2 \omega}(\tau) \propto \int E_{\text {bias }}(\vec{r})\left(\int E_{T H z}(t, \vec{r}) I_{\omega}^{2}(t+\tau, \vec{r}) d t\right) d^{3} \vec{r}
$$

In the above, the inner integral is a temporal cross-correlation between the $\mathrm{THz}$ transient and the square of the probe beam intensity at the space point $\vec{r}$, while the outer integral sums the contributions from each point of the interaction volume.

We now consider our case of a $800 \mathrm{~nm}, 90$ fs-long pulse in a focused Gaussian beam of waist $6.5 \mu \mathrm{m}$ and a Rayleigh range of $41 \mu \mathrm{m}$. The beam is focused in an uniform THz field restrained to a volume of thickness $L_{x}$ (inset in Fig. 2(a)). The maximum induced SH $I_{2 \omega \max }$ as a function of $L_{x}$ calculated with Eq. (3) is shown in Fig. 2(a). A single-cycle THz transient with frequency components in the range $0.05-3.0 \mathrm{THz}$ was used for the calculations. For $L_{x}$ shorter than the Rayleigh range, the interaction length between the $\mathrm{THz}$ wave and the probe is defined by $L_{x}$. In the opposite limit of $L_{x}$ longer than the Rayleigh range, $I_{2 \omega}$ saturates. The interaction length is then limited by the geometrical dimensions of the focus of the probe beam, as indicated by the quadratic dependence of $I_{\omega}$ in Eq. (3). This is valid as long as the wavelength of highest frequency components within the $\mathrm{THz}$ transient is shorter than the Rayleigh range of the probe beam. The response function $R(f)=I_{2 \omega}(f) / E_{T H z}(f)$, which describes the spectral response, has been evaluated for different widths of the $\mathrm{THz}$ region as shown in Fig. 2(b). For small $L_{x}$, the response function is monotonously decreasing with frequency. For larger $L_{x}$, the effects of positive and negative parts of the $\mathrm{THz}$ waveform can interfere leading to sharp dips in the response.

In Fig. 3(a), we show a 2D map of $\mathrm{THz}$ induced $\mathrm{SH}$ $I_{2 \omega}(t, z)$ as a function of the THz transient-probe delay time $t$ for different positions $z$ of the probe beam along the waveguide can be obtained. Two $\mathrm{THz}$ transients are visible in the plot: the first transient, centered at 5 ps time delay, is the incident pulse propagating inside the waveguide. The second
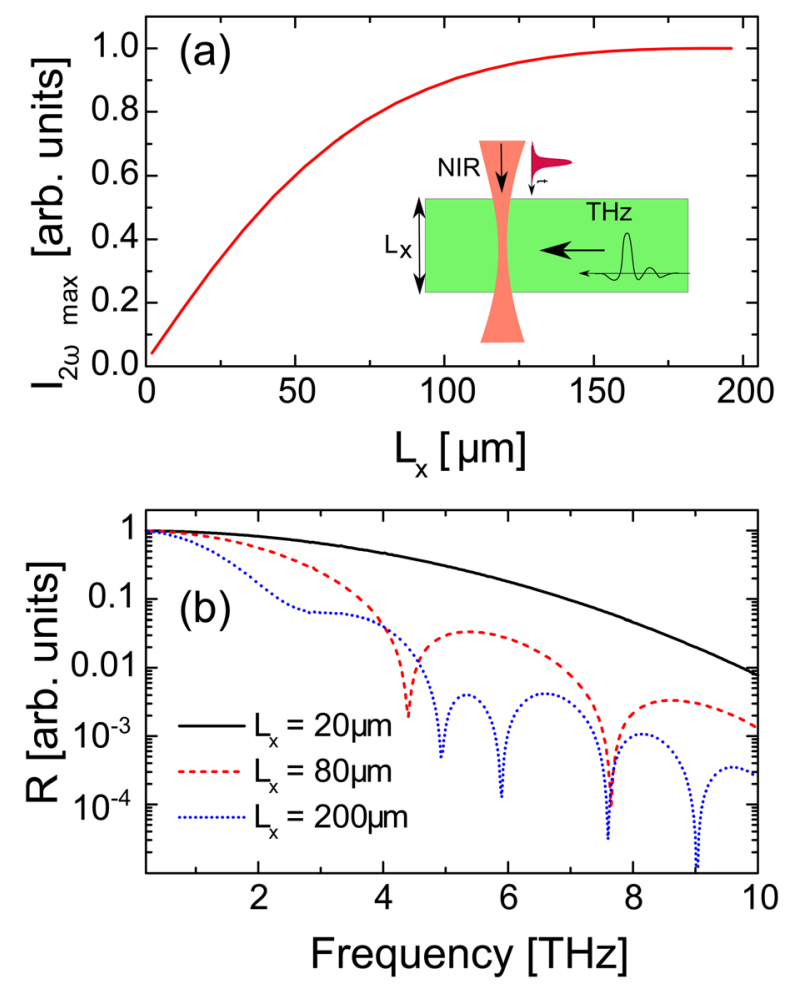

FIG. 2. (Color online) (a) Normalized maximum second harmonic intensity $I_{2 \omega}$ as a function of interaction length $L_{x}$ between fundamental and $\mathrm{THz}$ beams. The $800 \mathrm{~nm}$ beam waist at the focus point has been calculated to be $6.5 \mu \mathrm{m}$ and the Rayleigh range is $41 \mu \mathrm{m}$, pulse duration $90 \mathrm{fs}$. Inset presents considered geometry. (b) Normalized response function $R(f)$ for $L_{x}=20 \mu \mathrm{m}$, $80 \mu \mathrm{m}$, and $200 \mu \mathrm{m}$.

transient, propagating in the opposite direction, originates from the reflection at the waveguide end (position $z=0 \mathrm{~mm}$ ) due to the impedance mismatch between the waveguide $(z<0)$ and free space $(z>0)$. Values of $I_{2 \omega}(t, z)$ for $z<0$ has been corrected for the local bias field $E_{\text {bias }}(z)$, which changes with the plate separation. We have to note that for $z>0$ (outside the waveguide), the bias electric field quickly decays, which leads to vanishing $I_{2 \omega}(t, z)$. Fig. 3(b) shows the retrieved and simulated peak value of $\mathrm{THz}$ electric field as a function of NIR probe position $z$ along the waveguide. To calculate $E_{T H z}(t, z)$, the values of $I_{2 \omega}(t, z)$ have been corrected using the width of metal plates (and correspondingly by the interaction length $\left.L_{x}(z)\right)$ according to the dependence from Fig. 2(a). The time-domain numerical simulation was performed with CST Microwave Studio for a TPPWG made of aluminium treated as a lossy metal with conductivity of $\sigma=3.56 \cdot 10^{7} \mathrm{~S} / \mathrm{m}$. The simulation agrees well with the measurement except for $z>0$ where the fast decrease in the measured value is caused by the rapid decay of $E_{\text {bias }}$ outside the waveguide. The dip at $z=-0.15 \mathrm{~mm}$ has its origins in the interference between incident and reflected waves. The shape of the TPPWG structure can cause field enhancement in the proximity of the waveguide edges (propagating $\mathrm{THz}$ edge plasmons), ${ }^{9}$ while the calculated value of $E_{T H z}(t, z)$ is an integrated value of the $\mathrm{THz}$ electric field along the probe direction within the interaction volume. If additional freedom of the scanning stage movement along directions $x$ and $y$ is available, then edge enhancement effects can also be investigated using our method, with a transverse resolution determined by the Rayleigh range of the probe beam. 


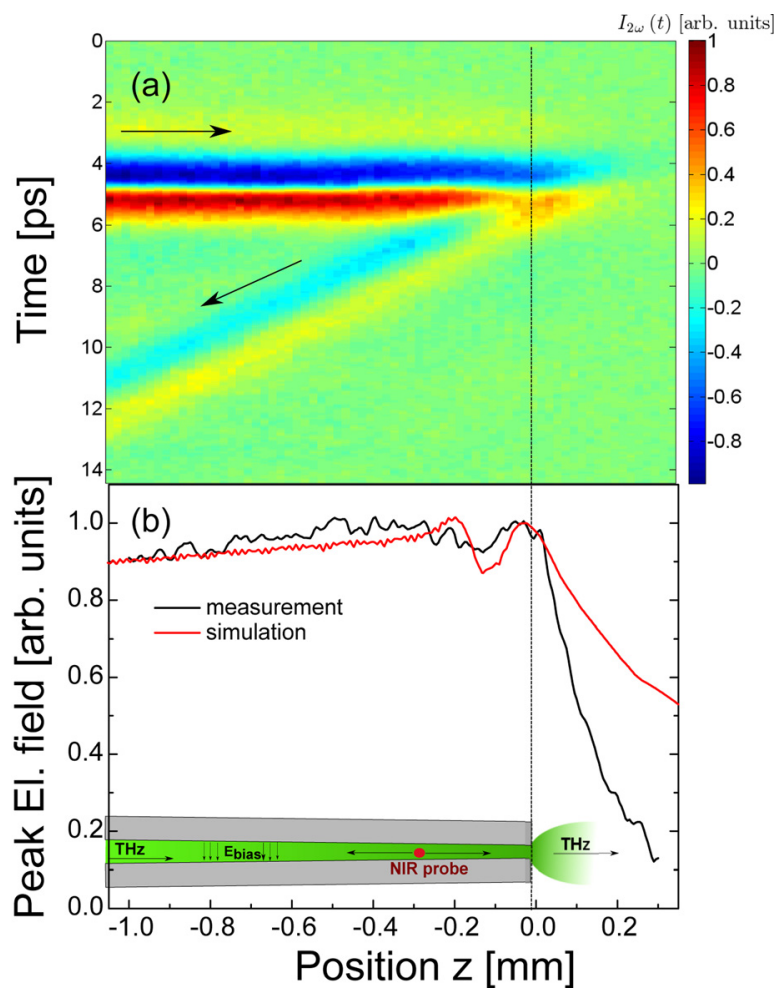

FIG. 3. (Color online) (a) 2D map of THz induced second harmonic $I_{2 \omega}(t)$ as a function of $\mathrm{THz}$ wave-probe delay time for different positions of the probe beam along the waveguide. Values of $I_{2 \omega}(t)$ have been corrected for the bias field. (b) Measured and simulated integrated peak THz electric field across the waveguide as a function of NIR probe position $z$.

Fig. 4 shows the measured frequency dependent amplitude reflection coefficient $\Gamma(f)=E_{\text {refl }}(f) / E_{\text {inc }}(f)$ at the waveguide end together with the result of a numerical simulation. For a good temporal separation between the incident and reflected pulses, the data (shown at the inset to the Fig. 4) are taken at a point $z=-0.9 \mathrm{~mm}$ from the waveguide end. That implies that the presented reflection coefficient $\Gamma$ includes not only reflection but also propagation effects, such as ohmic losses and wave scattering, which will lead to additional reduction of the amplitude of the reflected field. The reflection of the $\mathrm{THz}$ wave is caused by impedance mismatch between free space $Z_{0}=377$ $\Omega$ and the waveguide $Z_{P P W G}=\sqrt{(R+i \omega L) /(G+i \omega C)}$, where $R, L, G, C$ are correspondingly distributed resistance, inductance, conductance, and capacitance of the waveguide. We observe that the reflection coefficient $\Gamma=\left(Z_{\mathrm{PPWG}}-Z_{0}\right)$ / $\left(Z_{\text {PPWG }}+Z_{0}\right)$ decreases with increasing $\mathrm{THz}$ frequency. We find good agreement between measurement and simulation in the frequency range $0.1-0.5 \mathrm{THz}$. At higher frequencies, the measured reflection coefficient $\Gamma$ is smaller than theoretically predicted. This can be caused by the increased wave scattering

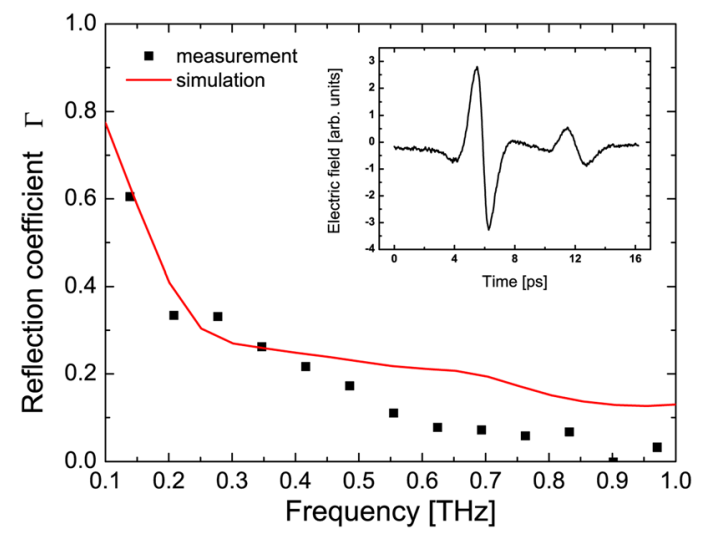

FIG. 4. (Color online) Measured and simulated frequency dependent amplitude reflection coefficient $\Gamma$ of the waveguide end. Waveform from point $z=-0.9 \mathrm{~mm}$ shown in the inset.

(especially important at higher frequencies and at interfaces) $)^{13}$ and increased ohmic losses at higher frequencies.

In conclusion, we have presented a non-invasive broadband method of imaging of the THz field inside PPWGs. The method does not disturb the propagating $\mathrm{THz}$ field as exemplified by the measurement of the $\mathrm{THz}$ reflection coefficient from the tip of the waveguide. The resolution of the method is restricted by the geometrical dimensions of the NIR probe focus spot only, while the frequency response depends on the interaction length between $\mathrm{THz}$ and the NIR probe.

We are grateful to Martin Nielsen and Frank Persson for manufacturing the tapered parallel plate waveguide and to the Idella Foundation, Danish Defense Acquisition and Logistics Organization, and Danish Research Council for Technology and Production Science NIM bus project for financial support.

${ }^{1}$ G. Gallot, S. P. Jamison, R. W. McGowan, and D. Grischkowsky, J. Opt. Soc. Am. B 17, 851 (2000).

${ }^{2}$ R. Mendis and D. Grischkowsky, Opt. Lett. 26, 846 (2001).

${ }^{3}$ R. Mendis and D. M. Mittleman, Opt. Express 17, 14839 (2009).

${ }^{4}$ J. S. Melinger, N. Laman, and D. Grischkowsky, Appl. Phys. Lett 93, 011102 (2008).

${ }^{5}$ D. G. Cooke and P. U. Jepsen, Phys. Status Solidi A 206, 997 (2009).

${ }^{6}$ M. I. Stockman, Phys. Rev. Lett. 93, 137404 (2004).

${ }^{7}$ A. Rusina, M. Durach, K. A. Nelson, and M. I. Stockman, Opt. Express 16, 18576 (2008).

${ }^{8}$ H. Zhan, R. Mendis, and D. M. Mittleman, Opt. Express 18, 9643 (2010).

${ }^{9}$ H. Zhan, R. Mendis, and D. M. Mittleman, J. Opt. Soc. Am. B 28, 558 (2011).

${ }^{10}$ J. Liu, R. Mendis, and D. M. Mittleman, Appl. Phys. Lett. 98, 231113 (2011).

${ }^{11}$ N. Karpowicz, J. Dai, X. Lu, Y. Chen, M. Yamaguchi, H. Zhao, X.-C. Zhang, L. Zhang, C. Zhang, M. Price-Gallagher, C. Fletcher, O. Mamer, A. Lesimple, and K. Johnson, Appl. Phys. Lett. 92, 011131 (2008).

${ }^{12}$ M. C. Hoffmann and J. A. Fulop, J. Phys. D: Appl. Phys. 44, 08300 (2011).

${ }^{13}$ S. Coleman and D. Grischkowsky, Appl. Phys. Lett. 83, 3656 (2003). 